\title{
Colonization of plant roots and enhanced atrazine degradation by a strain of Arthrobacter ureafaciens
}

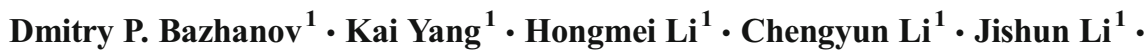 \\ Xiangfeng Chen ${ }^{2} \cdot$ Hetong Yang ${ }^{1}$
}

Received: 3 March 2017 / Revised: 10 June 2017 / Accepted: 20 June 2017 /Published online: 12 July 2017

(C) The Author(s) 2017. This article is an open access publication

\begin{abstract}
Our previous research found that culturable atrazine degraders associated with maize roots were dominated by genetically similar strains of Arthrobacter ureafaciens, suggesting their rhizosphere competence. The present study aimed to assess the root-colonizing capacity of strain A. ureafaciens DnL1-1 and to evaluate consequent rootassociated degradation of atrazine. A soil-sand assay and pot experiments provided evidence that $A$. ureafaciens DnL1-1 competitively colonized roots of maize, wheat, and alfalfa following seed inoculation. Atrazine was not absolutely required but promoted colonization of plant roots by the bacterium. In association with plants, A. ureafaciens DnL1-1 enhanced the degradation of atrazine and strongly reduced accumulation of its dealkylated metabolites. Our results show that after low-level inoculation of seeds, the bacterium A. ureafaciens DnL1-1 can establish root populations sufficient for the rapid degradation of atrazine in soil that makes it a promising bioremediation agent which can be easily applied to large areas of polluted soil. Application of the rootcolonizing, atrazine-degrading Arthrobacter bacteria as seed inoculants may be a reliable remediation strategy for soils
\end{abstract}

Dmitry P. Bazhanov and Kai Yang contributed equally to this work.

Electronic supplementary material The online version of this article (doi:10.1007/s00253-017-8405-3) contains supplementary material, which is available to authorized users.

Dmitry P. Bazhanov

bazhdp@outlook.com

1 Ecology Institute of Shandong Academy of Sciences, 19 Keyuan Road, Jinan 250014, Shandong Province, People's Republic of China

2 Shandong Provincial Analysis and Test Center of Shandong Academy of Sciences, Jinan, Shandong Province, People's Republic of China contaminated with chlorinated s-triazines and their degradation products.

Keywords Arthrobacter · Root colonization · Atrazine . Degradation products $\cdot$ Soil bioremediation $\cdot$ HPLC-MS/MS

\section{Introduction}

Strains belonging to the genus Arthrobacter are one of the most frequently isolated degraders of atrazine (Arbeli and Fuentes 2010; Krutz et al. 2010; Bazhanov et al. 2016). It is traditionally believed that Arthrobacter bacteria, being typical autochthonous soil microbes (Winogradsky 1925) and "K" strategists (Bowen 1980), are associated with bulk rather than rhizosphere soil (Cacciari and Lippi 1987). Nevertheless, some of the early (Cacciari and Lippi 1987) and many more recent investigations (Schweiger and Tebbe 2000; Duineveld et al. 2001; Gomes et al. 2001; Mansfeld-Giese et al. 2002; Polyanskaya et al. 2003; Costa et al. 2006; İnceoğlu et al. 2013) detected abundant populations of Arthrobacter bacteria in the rhizosphere of various plants. The observed prevalence of Arthrobacter spp. in root-associated microbial communities during the whole growth period or specific stages indicated their rhizosphere competence. However, few researches were done to clarify mechanisms required for rhizosphere growth of Arthrobacter strains. So, the hypothesis of efficient nutrient utilization by Arthrobacter bacteria during periods of lesser nutrient flux from roots (Herzberg et al. 1978) seemed to be the only trying to explain their rhizosphere-specific abundance.

Genomic analysis of atrazine-degrading Arthrobacter strains has shown that the high resistance to environmental stresses such as desiccation, oxidation and osmotic stress, adaptation to nutrient limitations due to metabolic versatility, 
and long survival time during starvation are the main mechanisms of their survival in soil (Mongodin et al. 2006; Dsouza et al. 2015). Nevertheless, the flagellar operon has been recently found in genome of atrazine-degrading isolate Arthrobacter sp. AK-YN10 (Sagarkar et al. 2016). The motility earlier observed in some bacteria of the genus Arthrobacter (Antheunisse 1974; Stanlake and Clark 1976) also suggests their active behavior in soil. Krutz et al. (2012) found that the density of atrazine-degrading bacteria in the rhizosphere was nearly twice of that in bulk soil. This suggests that the most successful soil degraders of atrazine are those associated with plant roots and therefore can be expected to actively colonize the rhizoplane and rhizosphere. Our previous research demonstrated that genetically similar atrazine-degrading Arthrobacter ureafaciens strains were ubiquitous in exposed to atrazine agricultural soils of Shandong Province and prevailed among atrazine degraders directly isolated from the maize rhizosphere (Bazhanov et al. 2016). The present study aimed to assess the root-colonizing capacity of strain A. ureafaciens DnL1-1 applied via inoculation of plant seeds and to evaluate consequent root-associated degradation of atrazine.

\section{Materials and methods}

\section{Soil}

Grassland soil S1 not adapted to atrazine was collected in East Jinan. Location and history of the site and characteristics of S1 soil were previously reported (Bazhanov et al. 2016).

\section{$s$-triazines}

Analytical grade atrazine (\#45330 Sigma-Aldrich, SaintLouis, MO, USA), atrazine-2-hydroxy (HA) (\#36631 Sigma-Aldrich, Saint-Louis, MO, USA), atrazine-desethyl (DEA) (\#36629 Sigma-Aldrich, Saint-Louis, MO, USA), atrazine-desisopropyl (DIA) (\#36628 Sigma-Aldrich, SaintLouis, MO, USA), and atrazine-desethyl-desisopropyl (DAA) (\#36667 Sigma-Aldrich, Saint-Louis, MO, USA) were used as calibration standards and to spike $\mathrm{S} 1$ soil samples in extraction recovery experiments. Deuterated atrazine-d5 $\left({ }^{2} \mathrm{H}_{5^{-}}\right.$ ATZ) (\#34053, Sigma-Aldrich, Saint-Louis, MO, USA), atrazine-2-hydroxy D5 ( ${ }^{2} \mathrm{H}_{5}$-HA) (\#DRE-XA10333100ME, LGC Standards, Teddington, UK), atrazine-desisopropyl D5/ ethylamino D5/ ( ${ }^{2} \mathrm{H}_{5}$-DIA) (\#DRE-C10332100, LGC Standards, Teddington, UK), desethylatrazine- $\mathrm{d}_{7} /$ iso-propyl$\mathrm{d}_{7} /\left({ }^{2} \mathrm{H}_{7}\right.$-DEA) (\#D-5639, C/D/N Isotopes Inc., PointeClaire, Canada), and ${ }^{13} \mathrm{C}$ atrazine-desethyl-desisopropyl $\left({ }^{13} \mathrm{C}_{3}\right.$-DAA) (\#CLM-7528-S, Cambridge Isotope Laboratories, Inc., Tewksbury, MA, USA) were used as internal standards at concentrations of $5 \mathrm{ppb}\left({ }^{2} \mathrm{H}_{5}\right.$-ATZ, ${ }^{2} \mathrm{H}_{5}$-HA,
${ }^{2} \mathrm{H}_{7}$-DEA $)$ and $20 \mathrm{ppb}\left({ }^{2} \mathrm{H}_{5}\right.$-DIA, ${ }^{13} \mathrm{C}_{3}$-DAA $)$. The standard stock solutions were prepared in HPLC-grade methanol (Fisher Scientific, Pittsburgh, USA).

Technical atrazine powder $(\geq 97 \%$; Shandong Dehao Chemical Co., Ltd., Weifang, China) was used in the routine growth media for atrazine-degrading bacteria, in soil-sand tube assay, and pot experiments. The high-performance liquid chromatography-tandem mass spectrometry (HPLC-MS/MS) analysis (performed in the manner described below) detected that the technical atrazine contained $0.078 \pm 0.002 \% \mathrm{HA}$, $0.150 \pm 0.003 \%$ DEA, $0.120 \pm 0.0007 \%$ DIA, and $0.010 \pm 0.0004 \%$ DAA.

\section{Bacterial strains and growth media}

Atrazine-degrading strain A. ureafaciens DnL1-1 harboring the genes $\operatorname{tr} z$ and $a t z B C$ was earlier isolated from the maize rhizosphere by a direct plating method (Bazhanov et al. 2016). The strain was deposited in the China General Microbiological Culture Collection Center (CGMCC) under the registration no. CGMCC 9667. The type strain A. ureafaciens CGMCC $1.1897^{\mathrm{T}}$ was obtained from the CGMCC. Mineral media SM and SMY (Bazhanov et al. 2016) were used to grow atrazine-degrading bacteria. Swimming motility was detected in liquid and semisolid ( $0.2 \%$ agar) medium SA, which contained salts of SM medium and $1 \mathrm{~g} / \mathrm{L}$ asparagine as sole carbon and nitrogen source.

\section{Degradation of atrazine and its metabolites by $A$. ureafaciens DnL1-1}

The strain A. ureafaciens DnL1-1 was grown in liquid medium SM to which stock solutions of analytical grade atrazine, DEA, DIA, and DAA were added separately or in combination instead of the routine atrazine stock suspension. The inoculum was prepared from 3-day cultures on SMY plates at $28{ }^{\circ} \mathrm{C}$. Ten colonies were suspended in $1 \mathrm{~mL}$ buffer (SM medium salt solution) and twice washed by centrifugation (3000 $\times \mathrm{g}, 3 \mathrm{~min})$. A $30 \mathrm{~mL}$ fresh medium in $250-\mathrm{mL}$ Erlenmeyer flasks was inoculated 1:1000 with the suspension and incubated at $28{ }^{\circ} \mathrm{C}$ without shaking. Growth of the cultures was monitored by plating on R2A agar (\#17209 SigmaAldrich, Saint-Louis, MO, USA). Samples for HPLC-MS/MS analyses were prepared by mixing equal volumes of culture and methanol followed by removing insoluble material by centrifugation $(8000 \times g, 2 \mathrm{~min})$. The mean mass and molar concentrations of the analytes and $95 \%$ confidence intervals were calculated based on the analyses of four replicate samples. For graphic representation, the mean colony forming unit (CFU) titers and molar concentrations were transformed to decimal logarithms (lg). 


\section{Motility tests}

To detect swimming motility, a $20 \mathrm{~mL}$ liquid medium SA in 250-mL Erlenmeyer flasks was inoculated 1:100 with a suspension of A. ureafaciens DnL1-1 cells and incubated at $28^{\circ} \mathrm{C}$ without shaking. The cultures were examined by light microscopy at $24 \mathrm{~h}$. For semisolid agar assay, a $2 \mu \mathrm{L}$ suspension of A. ureafaciens DnL1-1 was inoculated onto the center of SM and SA semisolid $(0.2 \%)$ agar plates. The plates were incubated at $28^{\circ} \mathrm{C}$ for 2 weeks. Migration of A. ureafaciens DnL11 along fungal hyphae was detected on soft $(0.5 \%$ agar $) \mathrm{SM}$ medium. A 5-mm plug from the culture of Rhizoctonia solani strain 21 (Rovira et al. 1986) on potato dextrose agar (PDA) was transferred to the center of an SM soft agar plate, which was incubated at $28^{\circ} \mathrm{C}$ for 2 days until the mycelium reached 20-22-mm diameter. A $10 \mu \mathrm{L}$ suspension of A. ureafaciens DnL1-1 was then inoculated onto the mycelium center (PDA plug). The plates were further incubated for additional 6 days.

\section{Seed inoculation}

A suspension of twice-washed cells of A. ureafaciens DnL1-1 was serially diluted from 1:30 to 1:1000 to prepare inocula with various cell densities. For the soil-sand assay, germinated non-sterilized seeds with radicles $\leq 1 \mathrm{~mm}$ were soaked in $30 \mathrm{~mL}$ of the diluted bacterial suspensions in Petri dishes for $1 \mathrm{~h}$. Control seeds were soaked in sterile buffer. The seeds were then carefully blotted on sterile filter paper, placed in a Petri dish, and immediately used for the assay.

To determine inoculum level on seeds, 10 wheat seeds or 20 alfalfa seeds were transferred into a $2-\mathrm{mL}$ polypropylene tube with $1 \mathrm{~mL}$ buffer, and five maize seeds into a $5-\mathrm{mL}$ polypropylene tube with $2.5 \mathrm{~mL}$ buffer. The tubes were secured in a MO BIO Vortex Adapter tube holder assembled on a Vortex-Genie ${ }^{\circledR} 2$ Vortex (MO BIO Laboratories, Inc., Carlsbad, CA, USA) and vortexed at maximum speed for $10 \mathrm{~min}$. The resulting suspensions and their serial dilutions were plated onto SM agar. Colonies of A. ureafaciens DnL11 were counted after 4 days at $28{ }^{\circ} \mathrm{C}$. Populations of indigenous seed bacteria were assessed in the non-inoculated control seeds by plating on R2A agar (Sigma-Aldrich, Saint-Louis, MO, USA). Bacterial densities and confidence intervals were calculated according to Koch (1981).

Dry wheat seeds were inoculated in a similar manner, airdried, and kept at room temperature. Seed population densities of A. ureafaciens DnL1-1 were assessed on the day of sowing.

\section{Soil-sand assay for assessment of root colonization and atrazine degradation}

The assay was performed in glass tubes $(25 \times 200 \mathrm{~mm})$ in the manner described by Scher et al. (1984) with modifications (Supplementary Fig. S1). Each tube contained $33 \mathrm{~g}$ of coarse sand moistened with $7 \mathrm{~mL}$ of $5 \mathrm{mM} \mathrm{KH_{2 }} \mathrm{PO}_{4}$ or atrazine solution in $5 \mathrm{mM} \mathrm{KH}_{2} \mathrm{PO}_{4}$ with a concentration of $0.5,2.5$, or $25.0 \mu \mathrm{g} / \mathrm{mL}$ to obtain herbicide doses of $3.5,17.5$, and $175.0 \mu \mathrm{g} /$ tube, respectively. The sand was overlaid with $2 \mathrm{~g}$ raw S1 soil adjusted to $18 \%$ moisture. One inoculated maize or wheat seed or three alfalfa seeds were added per tube. The seeds were covered with another $2 \mathrm{~g} \mathrm{~S} 1$ soil, closed with foil caps, and incubated in a HPG-400 BX growth chamber (Harbin Donglian Electronic Technology Development Co., Ltd., Harbin, China) at $26^{\circ} \mathrm{C}, 12,000 \mathrm{~lx}, 12$-h day/night cycle. No water was added to the tubes during incubation.

Root colonization was assessed 21-23 days post seeding. Plants were carefully removed from the tubes. All roots were excised under the soil and placed into Petri dishes with $30 \mathrm{~mL}$ buffer for $10 \mathrm{~min}$ to facilitate detachment of sand grains. Then, the roots were placed on filter paper for $2 \mathrm{~s}$ to remove excess moisture and transferred to 2-mL polypropylene tubes with $1 \mathrm{~mL}$ buffer. The tubes were secured in a MO BIO Vortex Adapter tube holder assembled on a Vortex-Genie ${ }^{\circledR} 2$ Vortex (MO BIO Laboratories, Inc., Carlsbad, CA, USA) and vortexed at maximum speed for $10 \mathrm{~min}$ to detach rootassociated bacteria. The resulting suspensions and their serial dilutions were plated onto SM agar. The washed root sections were blotted and immediately weighed. Mean lg CFU/g fresh root and $95 \%$ confidence intervals were calculated based on five replicate tubes for each treatment by using the descriptive statistics tool of MS Excel.

Atrazine degradation in the tubes was assessed 30 days post seeding. The tubes were closed with silicon plugs and stored at $-20{ }^{\circ} \mathrm{C}$. The extraction from the whole volume of soil-sand in a tube was performed in the manner described by Krutz et al. (2008). Atrazine was quantified by HPLC-MS/ MS, as described below.

\section{Assessment of root colonization and degradation of atrazine in a soil pot assay}

Soil S1 was passed through a 2-mm sieve and weighed (300 g dry weight equivalents) into $500-\mathrm{mL}$ polypropylene pots. Each pot received $70 \mathrm{~mL}$ of $5 \mathrm{mM} \mathrm{KH}_{2} \mathrm{PO}_{4}$ or solution of atrazine in $5 \mathrm{mM} \mathrm{KH}_{2} \mathrm{PO}_{4}$ at a concentration of $25.0 \mu \mathrm{g} / \mathrm{mL}$ to provide a dose of $1750 \mu \mathrm{g} /$ pot that was equivalent to $17.5 \mathrm{~kg} /$ ha on the basis of soil weight $3000 \mathrm{t} / \mathrm{ha}$. Seven inoculated or non-inoculated (control) dry wheat seeds were sown to each pot. Additional controls for assessment of atrazine degradation were pots with soil to which no seeds were sown and soil which received inoculated seeds with embryos removed. The pots were placed outdoors and incubated under sunlight with natural photoperiod of Jinan in September to October, day temperatures of $20-26{ }^{\circ} \mathrm{C}$ and $15-20{ }^{\circ} \mathrm{C}$ at night. During incubation, distilled water was added to the soil along the pot sides using a 10-mL Eppendorf pipette and sterile tips. 
Root colonization and degradation of atrazine were assessed 28 days post seeding. Roots were retrieved from the soil cores and cut $15 \mathrm{~mm}$ below the seeds. Loosely adhering soil was carefully removed by hand using sterile latex gloves. Then, the roots were processed and root-associated populations were evaluated as described in the "Soil-sand assay for assessment of root colonization and atrazine degradation" section. The soil samples were freeze-dried, homogenized, transferred into polyethylene bags, and kept at $-20{ }^{\circ} \mathrm{C}$. Atrazine and its degradation products were extracted from soil and analyzed by HPLC-MS/MS, as described below. Means and 95\% confidence intervals for the population densities and amounts of the analytes were calculated based on the results obtained from four replicate pots for each treatment by using the descriptive statistics tool of MS Excel. Molar concentrations of extractable atrazine and its transformation products were calculated to evaluate the total (atrazine plus degradation products) soil contamination.

\section{BOX-PCR genotyping}

Template DNAs and PCR mixtures were prepared as previously described (Bazhanov et al. 2016). The temperature program was as follows: $95^{\circ} \mathrm{C}$ for $2 \mathrm{~min}$; then 35 cycles at $94^{\circ} \mathrm{C}$ for $30 \mathrm{~s}, 60^{\circ} \mathrm{C}$ for $30 \mathrm{~s}$, and $72{ }^{\circ} \mathrm{C}$ for $2 \mathrm{~min}$; and final extension $72{ }^{\circ} \mathrm{C}$ for $10 \mathrm{~min}$. The amplification products were analyzed by gel electrophoresis as described before (Bazhanov et al. 2016).

\section{Extraction of atrazine and its degradation products from soil}

Atrazine and its degradation products were extracted from soil by the vortexing method. For extraction, $1 \mathrm{~g}$ samples of freeze-dried homogenized soil were weighed into 2-mL tubes and mixed with $1 \mathrm{~mL}$ methanol-water solution $(4: 1 \mathrm{v} / \mathrm{v})$. The tubes were secured horizontally in a MO BIO Vortex Adapter assembled on VortexGenie® 2 Vortex (MO BIO Laboratories, Inc., Carlsbad, CA, USA), vortexed at maximal speed for $1 \mathrm{~h}$, and left in the Vortex Adapter for $16 \mathrm{~h}$. After the incubation, tubes were vortexed for $5 \mathrm{~min}$. The suspension was centrifuged at $8000 \times \mathrm{g}$ for $3 \mathrm{~min}$, and the supernatant was transferred to a $10-\mathrm{mL}$ tube. Then, four additional extractions with $1 \mathrm{~mL}$ methanol/water were performed, consisting of $30 \mathrm{~min}$ vortexing and centrifugation at $8000 \times g$ for $3 \mathrm{~min}$. All vortexing steps and the incubation after the first vortexing were performed at $30{ }^{\circ} \mathrm{C}$. The supernatants were combined and analyzed by HPLC-MS/MS as described below.

To evaluate extraction efficiency, dry S1 soil was spiked with 10 and $200 \mathrm{ng} / \mathrm{g}$ each of atrazine, HA, DEA, DIA, and DAA. Blank samples of S1 soil were extracted to assess background contamination. The mean percent recoveries of the analytes and $95 \%$ confidence intervals at 10 and $200 \mathrm{ng} / \mathrm{g}$ were, respectively, $87.7 \pm 4.4$ and $94.6 \pm 5.9$ for atrazine,
$86.4 \pm 2.2$ and $86.6 \pm 2.8$ for HA, $93.7 \pm 3.8$ and $94.3 \pm 1.1$ for DEA, $95.6 \pm 2.7$ and $92.8 \pm 1.5$ for DIA, and $104.5 \pm 4.5$ and $99.8 \pm 5.7$ for DAA.

\section{HPLC-MS/MS}

The culture liquids and soil extracts were analyzed by HPLCMS/MS on an UltiMate 3000 HPLC system interfaced to a TSQ Vantage triple-quadrupole mass spectrometer (Thermo Fisher Scientific, Waltham, MA, USA). Atrazine and metabolites were separated on a Thermo Scientific ${ }^{\mathrm{TM}}$ Acclaim $^{\mathrm{TM}} 120$ C18 column $(250 \times 4.6 \mathrm{~mm}$, particle size $5 \mu \mathrm{m})$ with a flow rate of $0.6 \mathrm{~mL} / \mathrm{min}$. Methanol (A) and $0.1 \%$ aqueous formic acid solution (B) were used for HPLC gradient elution. The program of gradient elution was 0 to $6 \mathrm{~min}, 5 \% \mathrm{~A} ; 20$ to $25 \mathrm{~min}, 90 \% \mathrm{~A}$; and 25.1 to $30 \mathrm{~min}, 5 \% \mathrm{~A}$. The MS/MS analysis was performed in positive electrospray ionization mode and transitions were measured in multiple reaction monitoring. Parameters of MS/MS and the limits of detection and quantification for the analytes are specified in Table 1.

\section{Results}

\section{Degradation capacity of $A$. ureafaciens DnL1-1}

The atrazine-degrading capacity of A. ureafaciens DnL1-1 culture was assessed in SM medium containing $2 \mathrm{mg} / \mathrm{L}$ atrazine (analytical grade). Trace amounts of the herbicide $(\leq 96.0 \mathrm{ng} / \mathrm{L})$ were detected in the culture liquid $24 \mathrm{~h}$ after inoculation (Fig. 1 and Supplementary Table S1). At 48 h, atrazine was not detected in the medium. Nearly 14.0 molar percent of the degraded atrazine were transformed into HA, and the latter was slowly degraded during further incubation of the culture. After 25 days, HA was still detected at a concentration of $5.68 \pm 1.22 \mu \mathrm{g} / \mathrm{L}$, which was equivalent to nearly $0.3 \%$ of the initial molar content of atrazine. In the medium supplemented with DEA, DIA, and DAA, each at a concentration of $1.0 \mathrm{mg} / \mathrm{L}$, DEA was not detected after 3-day incubation of A. ureafaciens DnL1-1 culture (Fig. 1 and Supplementary Table S2). The content of DIA was reduced nearly 100 -fold (to a level of $9.97 \pm 1.57 \mu \mathrm{g} / \mathrm{L}$ ) during 4 days. After 21 days, DIA was still detected in the medium at a concentration of $2.26 \pm 0.20 \mu \mathrm{g} / \mathrm{L}$ (nearly $0.23 \%$ of the initial DIA content). No degradation of DAA by $A$. ureafaciens DnL1-1 was observed during 3-week incubation (Fig. 1 and Supplementary Table S2).

\section{Motility and migration of $A$. ureafaciens DnL1-1}

All tested 24-h cultures of A. ureafaciens DnL1-1 in liquid SA medium were rods, some of which exhibited rapid directional movement. The colony of A. ureafaciens DnL1-1 expanded in 
Table 1 MS/MS parameters and the limits of detection (LOD) and quantification (LOQ)

\begin{tabular}{|c|c|c|c|c|c|c|c|c|}
\hline $\begin{array}{l}\text { Analytes and internal } \\
\text { standards (IS) }\end{array}$ & $\begin{array}{l}\text { Retention } \\
\text { time (min) }\end{array}$ & $\begin{array}{l}\text { Precursor } \\
\text { ion }(m / z)\end{array}$ & $\begin{array}{l}\text { Product } \\
\text { ions }(\mathrm{m} / \mathrm{z})\end{array}$ & $\begin{array}{l}\text { Collision } \\
\text { energies }(\mathrm{eV})\end{array}$ & $\begin{array}{l}\text { Analytical LOD } \\
(\mathrm{ng} / \mathrm{mL})\end{array}$ & $\begin{array}{l}\text { Analytical LOQ } \\
(\mathrm{ng} / \mathrm{mL})\end{array}$ & $\begin{array}{l}\mathrm{LOD}^{\mathrm{a}} \text { in soil } \\
(\mathrm{ng} / \mathrm{g})\end{array}$ & $\begin{array}{l}\mathrm{LOQ}^{\mathrm{a}} \text { in soil } \\
(\mathrm{ng} / \mathrm{g})\end{array}$ \\
\hline Atrazine & 24.6 & 216.0 & $\begin{array}{r}174.0 \\
104.0 \\
68.0\end{array}$ & $\begin{array}{l}18 \\
29 \\
34\end{array}$ & $<0.01$ & 0.05 & $<0.045$ & 0.225 \\
\hline${ }^{2} \mathrm{H}_{5}$-ATZ (IS) & & 221.0 & 179.0 & 18 & $\mathrm{n} / \mathrm{a}$ & $\mathrm{n} / \mathrm{a}$ & $\mathrm{n} / \mathrm{a}$ & $\mathrm{n} / \mathrm{a}$ \\
\hline HA & 17.4 & 198.1 & $\begin{array}{r}156.0 \\
86.0 \\
69.0\end{array}$ & $\begin{array}{l}17 \\
23 \\
33\end{array}$ & $<0.01$ & 0.05 & $<0.045$ & 0.225 \\
\hline${ }^{2} \mathrm{H}_{5}-\mathrm{HA}$ (IS) & & 202.7 & $\begin{array}{r}160.9 \\
118.9 \\
69.8\end{array}$ & $\begin{array}{l}18 \\
23 \\
34\end{array}$ & $\mathrm{n} / \mathrm{a}$ & $\mathrm{n} / \mathrm{a}$ & $\mathrm{n} / \mathrm{a}$ & $\mathrm{n} / \mathrm{a}$ \\
\hline DEA & 21.7 & 188.0 & $\begin{array}{r}146.0 \\
104.0 \\
68.0\end{array}$ & $\begin{array}{l}17 \\
25 \\
30\end{array}$ & 0.02 & 0.1 & 0.09 & 0.45 \\
\hline${ }^{2} \mathrm{H}_{7}$-DEA (IS) & & 194.7 & $\begin{array}{r}146.8 \\
104.7 \\
79.8\end{array}$ & $\begin{array}{l}19 \\
26 \\
27\end{array}$ & $\mathrm{n} / \mathrm{a}$ & $\mathrm{n} / \mathrm{a}$ & $\mathrm{n} / \mathrm{a}$ & $\mathrm{n} / \mathrm{a}$ \\
\hline DIA & 20.0 & 174.0 & $\begin{array}{r}132.0 \\
104.0 \\
68.1\end{array}$ & $\begin{array}{l}16 \\
22 \\
29\end{array}$ & 0.2 & 0.5 & 0.9 & 2.25 \\
\hline${ }^{2} \mathrm{H}_{5}$-DIA (IS) & & 178.6 & $\begin{array}{r}136.8 \\
104.8 \\
68.8\end{array}$ & $\begin{array}{l}19 \\
25 \\
31\end{array}$ & $\mathrm{n} / \mathrm{a}$ & $\mathrm{n} / \mathrm{a}$ & $\mathrm{n} / \mathrm{a}$ & $\mathrm{n} / \mathrm{a}$ \\
\hline DAA & 15.1 & 146 & $\begin{array}{r}110.0 \\
104.0 \\
68.1\end{array}$ & $\begin{array}{l}16 \\
18 \\
27\end{array}$ & 0.1 & 0.2 & 0.45 & 0.90 \\
\hline${ }^{13} \mathrm{C}_{3}$-DAA (IS) & & 148.9 & 113.0 & 16 & $\mathrm{n} / \mathrm{a}$ & $\mathrm{n} / \mathrm{a}$ & $\mathrm{n} / \mathrm{a}$ & $\mathrm{n} / \mathrm{a}$ \\
\hline
\end{tabular}

n/a not applicable

${ }^{\text {a }}$ Indicated LODs and LOQs in soil could be improved by concentration of the extracts

semisolid (0.2\% agar) SA medium, reaching the plate edge within 14 days (Fig. 2). No active migration of A. ureafaciens DnL1-1 was detected in semisolid SM agar. On soft SM agar, strain DnL1-1 readily colonized hyphae of $R$. solan $i$ strain 21 following inoculation of the mycelium center (Fig. 2).

\section{Root colonization by A. ureafaciens DnL1-1 in a soil-sand assay}

Colonization of maize, wheat, and alfalfa roots by A. ureafaciens DnL1-1 was evaluated at high and low inoculum levels on seeds (Table 2). Indigenous bacterial populations of germinating seeds reached $8.2 \pm 0.1 \mathrm{lg}$ CFU per maize seed, $7.1 \pm 0.1 \mathrm{lg}$ CFU per seed of wheat, and $6.3 \pm 0.1 \mathrm{lg}$ CFU per alfalfa seed. Seed populations of A. ureafaciens DnL1-1 at high inoculum levels were nearly equal to or several times lower than the indigenous populations of maize and wheat seeds and did not exceed $1 \%$ of the total bacteria on alfalfa seeds (Table 2). At low inoculum levels, the density of A. ureafaciens DnL1-1 seed populations was reduced 1001000-fold compared to the high inoculum levels.

As a preliminary check for the presence of root-associated atrazine degraders, the root systems of 3-week-old wheat plants were placed on SM agar and incubated at $28^{\circ} \mathrm{C}$ for 2 weeks. Clearing halos near roots of the inoculated plants became visible along the entire root length on the third day (Fig. 3). No atrazine dissolution was detected near or under the roots of control non-inoculated plants during the whole observation period.

In the tubes containing $175 \mu \mathrm{g}$ atrazine, recovered populations of A. ureafaciens DnL1-1 were above $5 \mathrm{lg}$ CFU/g fresh root of maize and above $6 \mathrm{lg}$ CFU/g fresh root of wheat regardless of the inoculum level (Table 2). Lower population densities of A. ureafaciens DnL1-1 were observed on wheat roots at an atrazine dose of $3.5 \mu \mathrm{g} /$ tube. However, the differences detected were statistically significant $(P<0.05)$ only at the low seed inoculum level. The density of $A$. ureafaciens DnL1-1 population associated with alfalfa roots was over $5 \mathrm{lg}$ $\mathrm{CFU} / \mathrm{g}$ fresh root at the high inoculum level and an atrazine dose of $17.5 \mu \mathrm{g} /$ tube (Table 2). Inoculation of alfalfa seeds with more than 100-fold reduced density resulted in somewhat lower root populations of the atrazine degrader.

At zero dose of atrazine, stable colonization of maize and wheat roots by $A$. ureafaciens DnL1-1 was observed only after the high-density inoculation of seeds. However, population densities of A. ureafaciens DnL1-1 on the roots were nearly 100 times lower than those detected at the high atrazine dose. At the low inoculum levels and zero atrazine dose, rootassociated populations of A. ureafaciens DnL1-1 were close to the detection limit or even not detected in some tubes. 

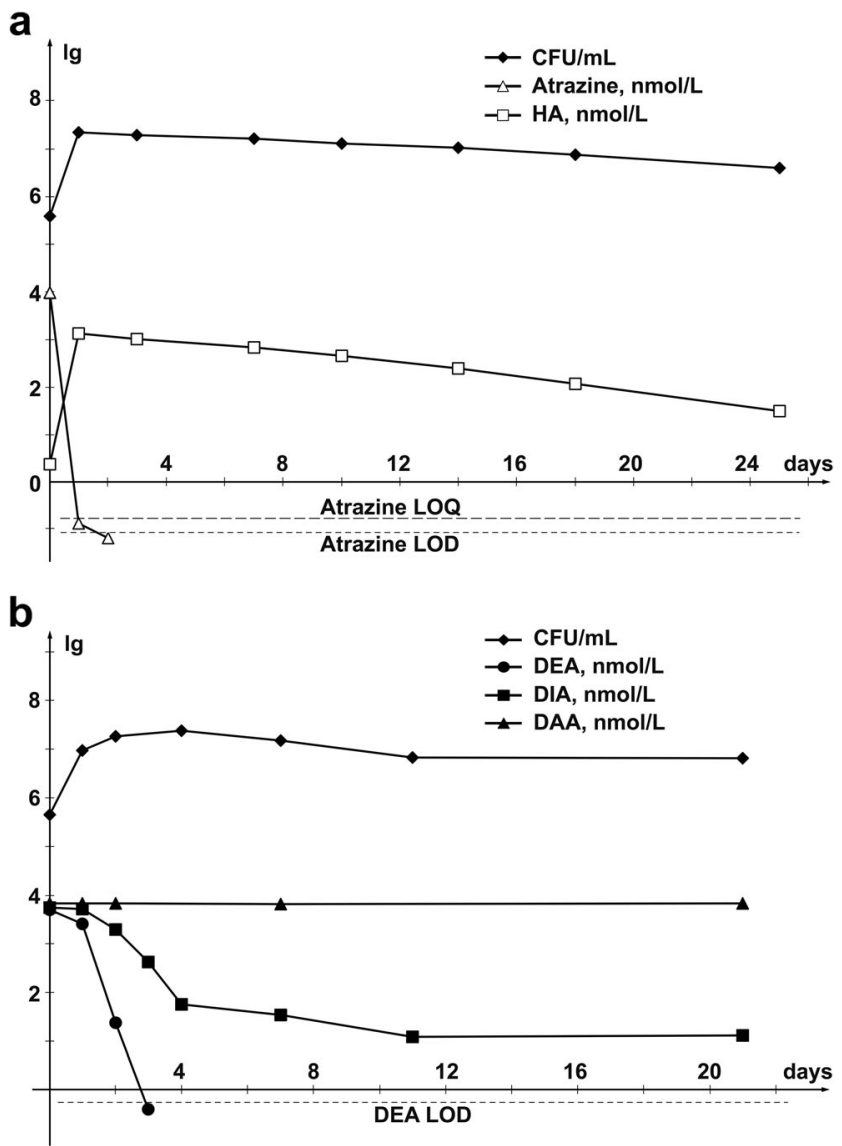

Fig. 1 Degradation of a atrazine, HA, and $\mathbf{b}$ dealkylated breakdown products by culture of $A$. ureafaciens DnL1-1

No atrazine degraders were detected on the roots of noninoculated control plants at any atrazine dose. The authenticity of atrazine-degrading colonies recovered from roots of the inoculated plants was examined by BOX-PCR. The banding patterns of all the isolates tested (a total of 56 independent isolates) were identical to those of A. ureafaciens DnL1-1 (Fig. 3).

\section{Degradation of atrazine by A. ureafaciens DnL1-1 in a soil-sand assay}

Atrazine damage became clearly visible in non-inoculated alfalfa and (at the $175 \mu \mathrm{g} /$ tube atrazine dose) wheat seedlings 20-24 days post seeding. Development of the injury symptoms resulted in plant death by the end of the incubation (Supplementary Fig. S1). The low (3.5 $\mu \mathrm{g} /$ tube) dose did not damage wheat plants incubated under artificial light in a growth chamber. No atrazine injury occurred in wheat plants inoculated by A. ureafaciens DnL1-1. The high inoculant dose eliminated atrazine injury symptoms in alfalfa, while slight growth inhibition still occurred after the low-level inoculation (Supplementary Fig. S1).

Analysis performed after 30 days of incubation demonstrated a profound effect of seed inoculation with A. ureafaciens DnL1-1 on atrazine degradation. At the high dose of the herbicide $(175.0 \mu \mathrm{g} / \mathrm{tube})$, its degradation by A. ureafaciens DnL1-1 in association with wheat roots was nearly complete (up to $99.8 \%$ ), regardless of the density of seed inoculation (Fig. 4 and Supplementary Table S3). Approximately $91.8 \%$ of atrazine were degraded by the wheatbacterium association in the tubes with low $(3.5 \mu \mathrm{g} /$ tube $)$ atrazine dose and high density of seed inoculation. As a result, the contents of residual atrazine in the tubes with inoculated wheat plants that had received the high and low herbicide doses were similar (less than $0.5 \mu \mathrm{g} /$ tube; Supplementary Table S3). Significantly higher residual contents of atrazine $(1.04 \pm 0.25 \mu \mathrm{g} /$ tube $)$ were measured at the low atrazine dose and low level of seed inoculum (Fig. 4 and Supplementary Table S3).

Up to $75.6 \%$ of the applied atrazine were degraded by A. ureafaciens DnL1-1 in association with alfalfa (Fig. 4 and Supplementary Table S3). No significant effect of the inoculation density on atrazine degradation was detected.

Atrazine degradation in the incubated controls ranged from 25.7 to $43.3 \%$ and was always significantly lower than in the inoculated tubes. At the high atrazine dose $(175.0 \mu \mathrm{g} /$ tube), its residues in the tubes with non-inoculated wheat plants were lower than in the control nonplanted tubes. No plant effect on the level of residual atrazine was detected at a dose of $3.5 \mu \mathrm{g} /$ tube for wheat and $17.5 \mu \mathrm{g} /$ tube for alfalfa.

\section{Colonization of wheat roots by $A$. ureafaciens DnL1-1 and degradation of atrazine in a soil pot assay}

The indigenous population on the dry wheat seeds averaged $2.28 \pm 0.05 \lg \mathrm{CFU} / \mathrm{seed}$ that was less than $1 \%$ of the inoculant
Fig. 2 Colony patterns of A. ureafaciens DnL1-1 in semisolid media a SM, b SA, and c migration of the bacterium along hyphae of $R$. solani strain 21
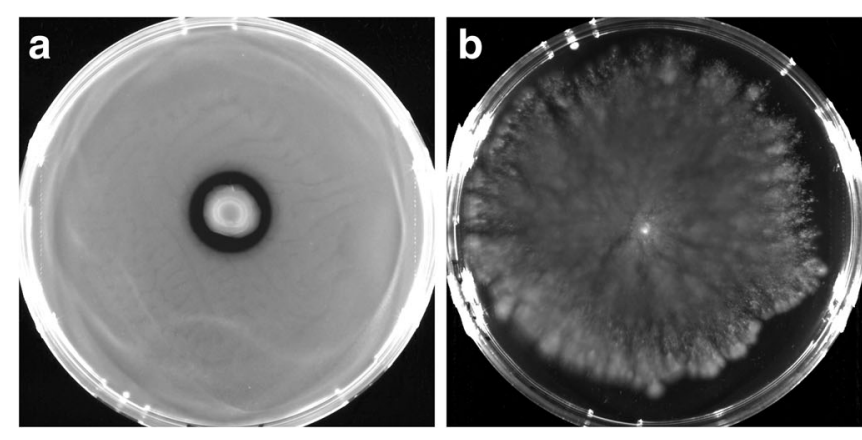
Table 2 Seed and root populations of $A$. ureafaciens DnL1-1 in soil-sand tube and soil pot assays

\begin{tabular}{|c|c|c|c|c|}
\hline Assay & Plant & Atrazine dose & $\begin{array}{l}\text { Seed population } \\
\text { (lg CFU/seed) }\end{array}$ & $\begin{array}{l}\text { Root population } \\
\text { (lg CFU/g fresh root) }\end{array}$ \\
\hline \multirow[t]{5}{*}{ Soil-sand tube } & Maize & $175.0 \mu \mathrm{g} /$ tube & $\begin{array}{l}\text { None } \\
4.23 \pm 0.03 \\
7.87 \pm 0.02 \\
\text { None } \\
4.23 \pm 0.03 \\
7.87 \pm 0.02\end{array}$ & $\begin{array}{l}<2.04 \\
<1.66-3.68 \\
3.32 \pm 0.86 \\
<2.0 \\
5.25 \pm 0.73 \\
5.88 \pm 0.51\end{array}$ \\
\hline & Wheat & None & $\begin{array}{l}\text { None } \\
3.45 \pm 0.04 \\
6.67 \pm 0.02\end{array}$ & $\begin{array}{l}<1.70 \\
<1.56-2.51 \\
4.43 \pm 0.50\end{array}$ \\
\hline & & $3.5 \mu \mathrm{g} / \mathrm{tube}$ & $\begin{array}{l}\text { None } \\
3.45 \pm 0.04 \\
6.67 \pm 0.02\end{array}$ & $\begin{array}{l}<2.46 \\
3.16 \pm 0.35 \\
4.95 \pm 0.97\end{array}$ \\
\hline & & $175.0 \mu \mathrm{g} / \mathrm{tube}$ & $\begin{array}{l}\text { None } \\
3.45 \pm 0.04 \\
6.67 \pm 0.02\end{array}$ & $\begin{array}{l}<2.07 \\
6.17 \pm 0.23 \\
6.26 \pm 0.41\end{array}$ \\
\hline & Alfalfa & $17.5 \mu \mathrm{g} /$ tube & $\begin{array}{l}\text { None } \\
2.08 \pm 0.04 \\
4.32 \pm 0.02 \\
\text { None } \\
2.08 \pm 0.04 \\
4.32 \pm 0.02\end{array}$ & $\begin{array}{l}<2.64 \\
<2.81 \\
<2.75-2.84 \\
<2.72 \\
4.59 \pm 0.56 \\
5.34 \pm 0.19\end{array}$ \\
\hline Soil pot & Wheat & $\begin{array}{l}\text { None } \\
1750 \mu \mathrm{g} / \text { pot }\end{array}$ & $\begin{array}{l}\text { None } \\
4.81 \pm 0.02 \\
\text { None } \\
4.81 \pm 0.02\end{array}$ & $\begin{array}{l}<1.70 \\
3.47 \pm 0.50 \\
<2.18 \\
6.04 \pm 0.24\end{array}$ \\
\hline
\end{tabular}

population (Table 2). Atrazine damage resulted in the death of all non-bacterized plants in control pots after 3-week incubation. Seed inoculation with A. ureafaciens DnL1-1 reduced herbicide damage and the bacterized wheat plants remained alive till the end of the experiment despite significant injury symptoms occurred. Twenty-eight days post seeding, root populations of $A$. ureafaciens DnL1-1 reached ca. $6 \mathrm{lg}$ CFU/g fresh root in the atrazine-supplemented soil and 10- to 100fold lower densities at zero atrazine dose (Table 2). All recovered atrazine degraders had similar colony morphology and produced BOX-PCR banding patterns similar to those of A. ureafaciens DnL1-1 (a total of 32 isolates were genotyped). No atrazine-degrading bacteria were detected on roots of control uninoculated plants both in the atrazine-supplemented and non-supplemented soils.

HPLC-MS/MS analysis revealed minor contamination of blank S1 soil with atrazine, HA, DEA, DIA, and DAA (Table 3). The atrazine content in atrazine-supplemented S1
Fig. 3 Recovery of A. ureafaciens DnL1-1 from wheat roots. a Roots of a control uninoculated plant. b A zone of atrazine degradation (clearing halo) in SM agar near roots colonized by $A$. ureafaciens DnL1-1 and c recovery of atrazine-degrading colonies. d BOX-PCR banding patterns of the recovered colonies. Lanes $T$ patterns of the type strain A. ureafaciens CGMCC $1.1897^{\mathrm{T}}$. Lanes I patterns of the inoculant strain A. ureafaciens DnL1-1. Lanes $M$ contain a DL 5000 DNA marker (TaKaRa Bio Inc.)
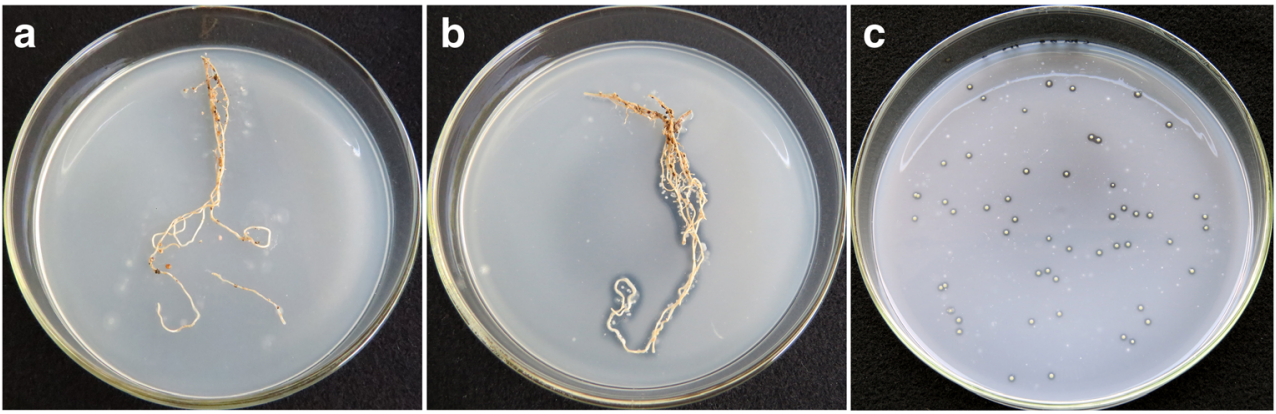

d
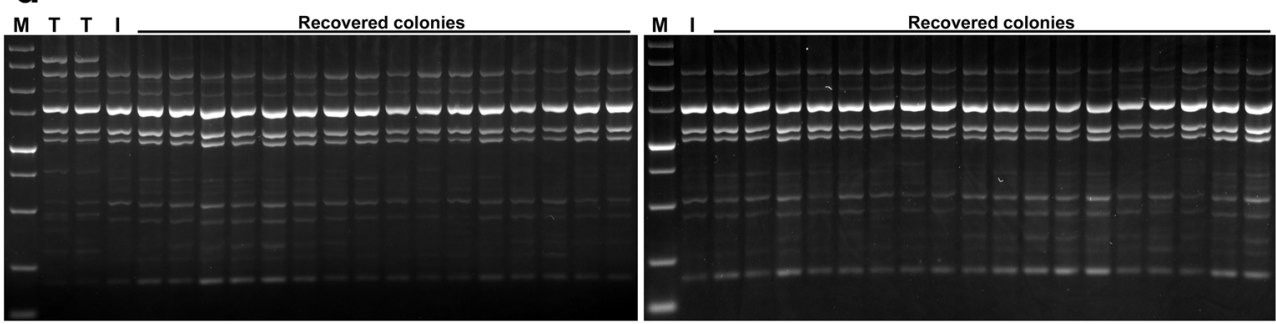


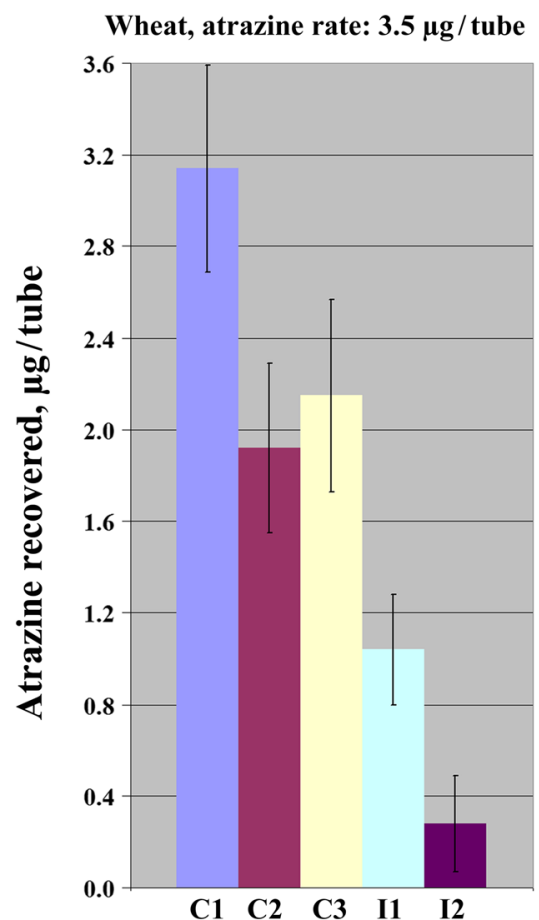

Fig. 4 Root-associated degradation of atrazine by A. ureafaciens DnL1-1 in a soil-sand tube assay. Inoculum levels $3.45 \pm 0.04 \mathrm{lg}$ CFU/seed (I1), $6.67 \pm 0.02 \mathrm{lg}$ CFU/seed (I2), $2.08 \pm 0.04 \mathrm{lg}$ CFU/seed (I3), $4.32 \pm 0.02 \mathrm{lg}$

soil averaged $5911.4 \pm 518.0 \mu \mathrm{g} / \mathrm{kg}$, indicating that the extraction recovery was $101.3 \pm 8.9 \%$. The concentrations of HA, DIA, and DEA in the atrazine-supplemented S1 soil exceeded background levels by nearly 70,80 , and 130 times, respectively, and the content of DAA was about twice the background level. While the amount of DAA measured was close to the expected level (a sum of DAA in blank soil and DAA in atrazine dose applied), the concentrations of HA, DIA, and DEA were much higher than expected (Table 3).

After 28-day incubation, the degradation of atrazine in nonplanted and planted control soils amounted to $42-46 \%$ (Table 3). The analysis of atrazine metabolites in the control soils revealed accumulation of DEA as a major breakdown product and HA, DIA, and DAA as minor ones. Seed

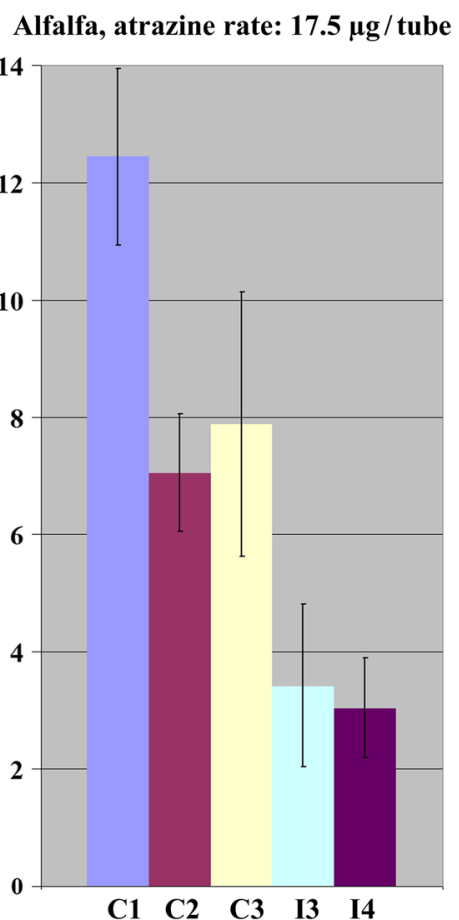

CFU/seed (I4). Controls: not incubated tubes (C1), not inoculated not planted tubes (C2), not inoculated planted tubes (C3)

Table 3 Quantification of atrazine and its degradation products in soil

\begin{tabular}{|c|c|c|c|c|c|c|c|c|}
\hline \multirow[t]{2}{*}{ Analyte } & \multirow{2}{*}{$\begin{array}{l}\text { Dose applied } \\
\text { (ng/g dry } \\
\text { soil) }\end{array}$} & \multicolumn{7}{|c|}{ Analyte concentrations in soils (ng/g dry soil) } \\
\hline & & Blank soil & $\begin{array}{l}\text { Blank soil + } \\
\text { atrazine } \\
\text { (expected) }\end{array}$ & $\begin{array}{l}\text { Blank soil + } \\
\text { atrazine } \\
\text { (measured) }\end{array}$ & $\begin{array}{l}\text { Uninoculated } \\
\text { unplanted } \\
\text { control }\end{array}$ & $\begin{array}{l}\text { Uninoculated } \\
\text { planted (wheat) } \\
\text { control }\end{array}$ & $\begin{array}{l}\text { Inoculated } \\
\text { unplanted } \\
\text { control }\end{array}$ & $\begin{array}{l}\text { A. ureafaciens DnL1-1 } \\
\text { in association with } \\
\text { wheat }\end{array}$ \\
\hline Atrazine & 5833.3 & $0.99 \pm 0.13$ & 5834 & $5911.4 \pm 518.0$ & $3192.7 \pm 387.5$ & $3404.7 \pm 272.7$ & $2404.1 \pm 480.5$ & $125.1 \pm 8.4$ \\
\hline HA & $4.55 \pm 0.12$ & $0.98 \pm 0.05$ & 5.55 & $67.7 \pm 2.8$ & $198.3 \pm 15.0$ & $226.1 \pm 44.4$ & $166.6 \pm 19.6$ & $151.7 \pm 8.6$ \\
\hline DEA & $8.75 \pm 0.18$ & $0.47 \pm 0.04$ & 9.25 & $63.2 \pm 1.5$ & $1029.2 \pm 114.6$ & $975.4 \pm 60.5$ & $440.1 \pm 36.9$ & $17.2 \pm 2.3$ \\
\hline DIA & $7.00 \pm 0.04$ & $0.25 \pm 0.01$ & 7.25 & $20.6 \pm 0.9$ & $219.0 \pm 18.5$ & $234.3 \pm 11.6$ & $122.3 \pm 12.3$ & $17.6 \pm 1.6$ \\
\hline DAA & $0.58 \pm 0.02$ & $0.42 \pm 0.08$ & 1.00 & $0.90 \pm 0.03$ & $186.8 \pm 15.6$ & $178.1 \pm 6.9$ & $60.9 \pm 9.1$ & $41.3 \pm 1.8$ \\
\hline
\end{tabular}

inoculation with A. ureafaciens DnL1-1 and the resultant colonization of roots led to the degradation of nearly $97.9 \%$ of the herbicide applied, prevented the accumulation of DIA, and reduced DEA concentration by 3.7 times compared to its level in the non-incubated atrazine-supplemented soil. The accumulation of DAA in soil that harbored bacterized wheat plants was reduced by more than four times, and the content of HA was reduced by nearly $25-33 \%$ compared to the incubated non-planted and planted controls.

Only about $59 \%$ of the atrazine were degraded in soil that received the inoculum of $A$. ureafaciens DnL1-1 on the nonviable wheat seeds (Table 3), while the contents of DEA, DIA, and DAA were higher by 25,7 , and 1.5 times, respectively, compared to those in soil with inoculated wheat plants. The 
content of HA in the inoculated planted and plantless soils was similar.

The total molar amount of extractable atrazine and its degradation products in the uninoculated non-planted and planted control soils reached $23.84 \pm 2.71$ and $24.72 \pm 1.96 \mu \mathrm{mol} / \mathrm{kg}$, respectively, or about $85 \%$ of their initial total amount $(28.21 \pm 2.43 \mu \mathrm{mol} / \mathrm{kg})$ in the atrazine-supplemented soil (Fig. 5 and Supplementary Table S4). The total contamination of soil that harbored A. ureafaciens DnL1-1 in association with wheat plants was $1.83 \pm 0.12 \mu \mathrm{mol} / \mathrm{kg}$ or nearly $6.5 \%$ of the initial level. The total yield of the analytes from the soil to which A. ureafaciens DnL1-1 was delivered on the nonviable seeds amounted to $15.52 \pm 2.63 \mu \mathrm{mol} / \mathrm{kg}$ (nearly $55 \%$ of the initial level).

\section{Discussion}

It is known that the ability to competitively grow on roots in the presence of soil microbes is a key criterion to recognize the root-colonizing bacteria (Kloepper and Beauchamp 1992). Therefore, the availability of a method for differentiating the introduced bacterium from indigenous microorganisms is essential for measuring its root-colonizing capacity. Unlike other known media for the selective isolation of atrazine degraders, SM agar enables their rapid direct recovery from field soil (Bazhanov et al. 2016), thus making possible the quantification of A. ureafaciens DnL1-1 in microbial communities harboring no other atrazine-degrading bacteria capable of growing on this medium.

This study provides evidence that A. ureafaciens strain DnL1-1 competitively colonizes plant roots following

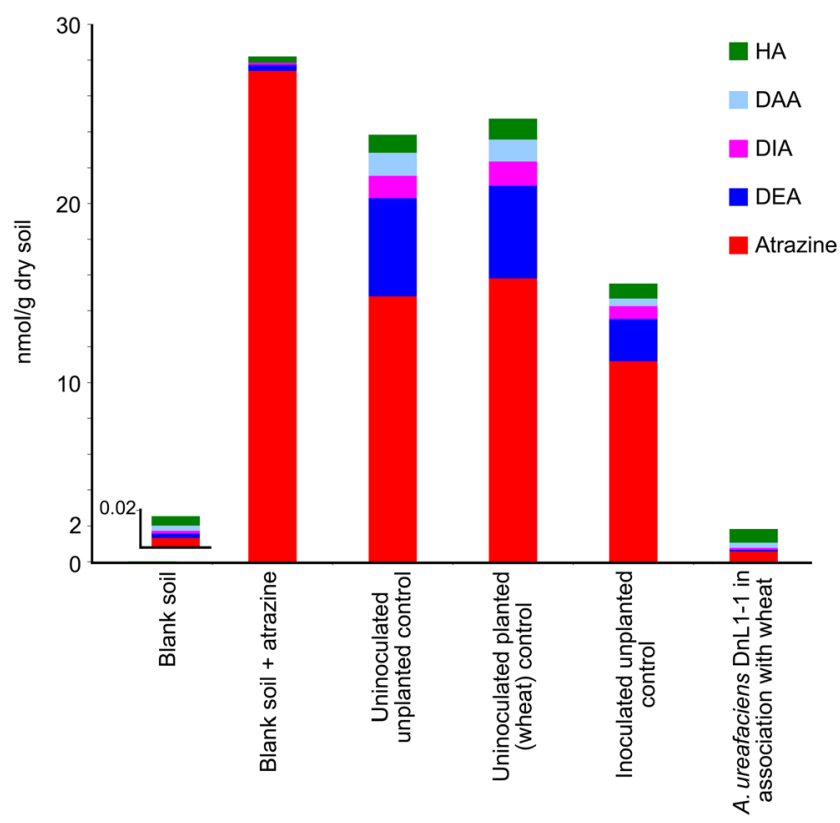

Fig. 5 Molar balance of atrazine and its degradation products in soil inoculation of seeds. Clearing halos produced in SM agar near the roots indicated that $A$. ureafaciens DnL1-1 migrated to the entire root system. In the soil-sand assay, the bacterium was recovered from roots of maize, wheat, and alfalfa, showing no strong "host" specificity. Scher et al. (1984) demonstrated that the assay enabled consistent assessment of the root-colonizing capacity due to competition of the test bacterium with indigenous soil microorganisms. Because no water is added to tubes during a soil-sand assay, the passive transfer of inoculant cells with the water flow is minimized, and active motion is required for migration of the tested bacteria on roots.

At the high inoculum levels and zero dose of atrazine, the densities of $A$. ureafaciens DnL1-1 root populations were within the range reported by Scher et al. (1984) for strains of fluorescent pseudomonads tested in a soil-sand assay under similar inoculation parameters and incubation conditions. This suggests that the root-colonizing capacity of A. ureafaciens DnL1-1 is comparable to those of fluorescent pseudomonads considered to be excellent root colonizers (Lugtenberg et al. 2001). Root populations of A. ureafaciens DnL1-1 at zero atrazine dose and low-level seed inoculation were close to the detection limit, indicating that the initial relative abundance of the introduced bacterium was critical for its rhizosphere growth. Such a result was not unexpected, considering large populations of indigenous seed and soil bacteria and assuming that a large portion of these were rhizosphere competent. A direct positive relationship between inoculum size on seeds and root population density was demonstrated for root-colonizing strains of fluorescent pseudomonads (Bull et al. 1991; Raaijmakers and Weller 2001); therefore, higher doses of inoculant on seeds were required to maintain root populations.

An atrazine dose as low as $3.5 \mu \mathrm{g} /$ tube facilitated the colonization of wheat roots by A. ureafaciens DnL1-1 following low-level seed inoculation, although the density of root populations of the colonizer still depended on the inoculum size. At the high atrazine dose, A. ureafaciens DnL1-1 reached the highest root populations similarly at both high and low levels of seed inoculation. The ability to establish root populations of a high density regardless of the density of seed population was so far found only in superior root colonizers (Raaijmakers and Weller 2001). The root-colonizing capacity of $A$. ureafaciens DnL1-1 observed in the pot experiment was similar to that determined in the soil-sand assay. Thus, both assays provided evidence that atrazine enhanced the competitiveness of A. ureafaciens DnL1-1 in the rhizosphere.

Numerous investigations have demonstrated that motility and chemotaxis are essential for bacterial colonization of plant roots (Lugtenberg et al. 2001; Compant et al. 2010). Our results clearly indicate that $A$. ureafaciens strain DnL1-1 exhibits swimming motility and chemotaxis toward asparagine. The observed migration along fungal hyphae is an example of A. ureafaciens DnL1-1 active behavior. The active migration 
may be also important for colonization of plant roots by A. ureafaciens DnL1-1. Studying synthesis of flagella in Arthrobacter atrocyaneus, Arthrobacter citreus, and Arthrobacter symplex, Stanlake and Clark (1976) found that motility was repressed in coccoidal cells for all the species and began only after induction to the rod morphology. Therefore, at least two mechanisms can be proposed by which atrazine favors the colonization of roots by A. ureafaciens DnL1-1. It is clear that the nutritional gains from catabolism of atrazine could provide a selective advantage to A. ureafaciens DnL11 in the rhizosphere, directly promoting growth of the population. In its turn, the activation of A. ureafaciens DnL1-1 cell growth might induce production of the motile rods, thus facilitating migration of the bacterium on roots.

Describing Bowen's (1980) "K" and "r" strategies of bacterial survival in soil, Lynch (1984) suggested that “... the completely successful soil organism will certainly exhibit characteristics of both types of ecological adaptation." The recently observed influence of plant species and/or growth stage on the rhizosphere abundance of Arthrobacter bacteria (Costa et al. 2006; İnceoğlu et al. 2013; Schweiger and Tebbe 2000) indicates their fast response to changing environmental conditions and suggests that arthrobacter's secrets of soil survival are not limited to the K strategy and stress tolerance mechanisms. Our study provides support to the idea that active behavior and zymogenous response can contribute to the ecological success of Arthrobacter. In the absence of atrazine, strain A. ureafaciens DnL1-1 competitively colonized plant roots, demonstrating spatial distribution although still remaining a minor component of the rhizosphere microbial community. In atrazine-supplemented soil, A. ureafaciens DnL1-1 established large root populations regardless of the inoculum size, exhibiting strong traits of the $r$ strategist.

The analysis of atrazine residues after growth of bacterized root systems provided evidence that inoculation of plant seeds with A. ureafaciens DnL1-1 had a substantial effect on the fate of the herbicide by enhancing its degradation. In a soil-sand assay with wheat plants, the amount of atrazine was reduced to less than $0.5 \mu \mathrm{g} /$ tube in all treatments where seed inoculation resulted in the establishment of large (ca. 5 to $6 \lg$ CFU/g root) populations of A. ureafaciens DnL1-1. The content of residual atrazine was significantly higher $(1.04 \pm 0.25 \mu \mathrm{g} /$ tube $)$ where the degrader's populations on wheat roots were lower than $4 \mathrm{lg} \mathrm{CFU} / \mathrm{g}$, indicating that $A$. ureafaciens DnL1-1 played a key role in atrazine degradation. Association of A. ureafaciens DnL1-1 with alfalfa plants also exhibited enhanced degradation of atrazine. However, despite successful colonization of alfalfa roots by strain DnL1-1, the level of atrazine residues was significantly higher than that detected in tubes which harbored wheat-A. ureafaciens DnL1-1 associations, indicating that the "host" plant affected the extent of atrazine degradation.
Root populations established by A. ureafaciens DnL1-1 on wheat in the soil pot assay were sufficient to degrade almost all of the atrazine applied to soil. In contrast, the level of atrazine residues in soil received A. ureafaciens DnL1-1 on non-viable wheat seeds was significantly higher and comparable to that in the control non-inoculated soil. This indicated that the high atrazine-degrading activity of A. ureafaciens DnL1-1 was associated with plant roots.

In both the soil-sand and soil assays, a significant portion of atrazine (42-46\%) was degraded in the control planted and plantless treatments not inoculated with $A$. ureafaciens DnL11. The $\mathrm{S} 1$ soil used in the soil-sand assay and pot experiments had not been exposed to atrazine, and no bacteria capable of rapid atrazine degradation could be isolated from it either directly or after enrichment (Bazhanov et al. 2016). The observed accumulation of DEA as a major degradation product and DIA and DAA as minor ones in the control treatments clearly demonstrated degradation of atrazine through the dealkylation pathway intrinsic to $s$-triazine non-adapted soils (Krutz et al. 2010). As a result, the total contamination by atrazine and its dealkylated products was only slightly reduced.

Seed inoculation and subsequent colonization of roots by A. ureafaciens DnL1-1 enhanced the degradation of atrazine and changed the formation of atrazine metabolites in soil. HA was the main degradation product detected in the soils harbored wheat-A. ureafaciens DnL1-1 association; however, its accumulation was reduced compared to the control noninoculated treatments. The contents of DEA and DIA were below their initial levels measured in the atrazinesupplemented S1 soil and the accumulation of DAA was reduced. Because no DAA-degrading activity was detected in pure culture of $A$. ureafaciens DnL1-1, the latter suggested that rapid degradation of atrazine by the introduced bacterium prevented its dealkylation by indigenous microorganisms.

Thus, the results of our study provide evidence that the root-colonizing capacity of atrazine-degrading strain A. ureafaciens DnL1-1 facilitates fast adaptation of planted soil to the herbicide. It is known that the efficacy of weed control by $s$-triazine herbicides can be significantly reduced in the adapted soils (Krutz et al. 2010). Almost complete elimination of atrazine injury symptoms in wheat and alfalfa observed in the soil-sand assay under artificial light and prevention of wheat seedling death in the pot experiment, where sunlight enhanced atrazine toxicity, indicated that inoculation of seeds with A. ureafaciens DnL1-1 significantly reduced plant exposure to the herbicide. The observed wide "host" range of the strain suggested that colonization of weed roots by atrazine-degrading A. ureafaciens might contribute to their resistance to the herbicide in field. On the other hand, seed inoculation followed by colonization of plant roots is an effective method of delivering A. ureafaciens 
DnL1-1 to contaminated soil. It is known that application of $s$-triazine-degrading bacteria for soil bioremediation requires high doses (from at least 5 to $7 \mathrm{lg} \mathrm{CFU} / \mathrm{g}$ dry soil) of inoculants (Topp 2001; Morgante et al. 2010; Wang et al. 2013; Sagarkar et al. 2016), repeated treatments (Newcombe and Crowley 1999; Morgante et al. 2010), and addition of carbon and energy sources to the soil for stimulation of the atrazine-degrading activity (Mandelbaum and Wackett 1996). Owing to its rootcolonizing ability, A. ureafaciens DnL1-1 can be introduced to atrazine-contaminated soil by a single low-dose treatment of seeds and can establish root populations sufficient to effectively degrade atrazine and reduce concentrations of its dealkylated metabolites. This makes strain A. ureafaciens DnL1-1 a promising bioremediation agent which can be easily applied to large areas of polluted soil.

The study reported here is the first to demonstrate the root-colonizing ability in an atrazine-degrading soil bacterium. Selective advantages from utilization of atrazine enhanced the root-colonizing capacity of the investigated strain A. ureafaciens DnL1-1, but were not absolutely required for the competitive colonization of plant roots. Our findings provide evidence that $A$. ureafaciens DnL1-1 can rapidly respond to favorable environmental conditions and establish large root populations after the low-level inoculation, indicating that the $r$ strategy of adaptation to soil environment and active behavior are intrinsic to at least some representatives of the genus Arthrobacter and can contribute to their environmental success. The ability to colonize plant roots and high atrazine-degrading capacity in soil makes A. ureafaciens DnL1-1 a promising model for studying agronomic and ecological implications of the root-associated atrazine degradation. The release of A. ureafaciens DnL1-1 through inoculation of plant seeds is a convenient method to deliver the atrazine degrader to large areas of contaminated soil.

Acknowledgements This work was supported by grants WQ20123700079 from One Thousand Talents Plan of China, tshw20120743 from Taishan Scholar Plan of Shandong Province, 2014QN021 from Youth Foundation of Shandong Academy of Sciences, and BS2014SW030 from Science Foundation for Distinguished Young Scholars of Shandong Province and Agricultural Technology grant from the Shandong Provincial Department of Agriculture. We thank Paul Harvey for providing the $R$. solani strain 21 and Maarten Ryder for critically reading this manuscript.

\section{Compliance with ethical standards}

Conflict of interest The authors declare that they have no conflict of interest.

Ethical approval This article does not contain any studies with human participants or animals performed by any of the authors.
Open Access This article is distributed under the terms of the Creative Commons Attribution 4.0 International License (http:// creativecommons.org/licenses/by/4.0/), which permits unrestricted use, distribution, and reproduction in any medium, provided you give appropriate credit to the original author(s) and the source, provide a link to the Creative Commons license, and indicate if changes were made.

\section{References}

Antheunisse J (1974) Motility and flagellation of Arthrobacter strains. Can J Microbiol 20:1411-1414

Arbeli Z, Fuentes C (2010) Prevalence of the gene $\operatorname{trz} N$ and biogeographic patterns among atrazine-degrading bacteria isolated from 13 Colombian agricultural soils. FEMS Microbiol Ecol 73:611-623

Bazhanov DP, Li C, Li H, Li J, Zhang X, Chen X, Yang H (2016) Occurrence, diversity and community structure of culturable atrazine degraders in industrial and agricultural soils exposed to the herbicide in Shandong Province, P.R. China. BMC Microbiol 16: 265. doi:10.1186/s12866-016-0868-3

Bowen GD (1980) Misconceptions, concepts and approaches in rhizosphere biology. In: Ellwood DC, Hedger JN, Latham MJ, Lynch JM, Slater JH (eds) Contemporary microbial ecology. Academic Press, London, pp 283-304

Bull CT, Weller DM, Thomashow LS (1991) Relationship between root colonization and suppression of Gaeumannomyces graminis var. tritici by Pseudomonas fluorescens strain 2-79. Phytopathology 81:954-959

Cacciari I, Lippi D (1987) Arthrobacters: successful arid soil bacteria: a review. Arid Soil Res Rehabil 1:1-30

Compant S, Clément C, Sessitsch A (2010) Plant growth-promoting bacteria in the rhizo- and endosphere of plants: their role, colonization, mechanisms involved and prospects for utilization. Soil Biol Biochem 42:669-678

Costa R, Götz M, Mrotzek N, Lottmann J, Berg G, Smalla K (2006) Effects of site and plant species on rhizosphere community structure as revealed by molecular analysis of microbial guilds. FEMS Microbiol Ecol 56:236-249

Dsouza M, Taylor MW, Turner SJ, Aislabie J (2015) Genomic and phenotypic insights into the ecology of Arthrobacter from Antarctic soils. BMC Genomics 16:36. doi:10.1186/s12864-015-1220-2

Duineveld BM, Kowalchuk GA, Keijzer A, van Elsas JD, van Veen JA (2001) Analysis of bacterial communities in the rhizosphere of chrysanthemum via denaturing gradient gel electrophoresis of PCRamplified 16SrRNA as well as DNA fragments coding for $16 \mathrm{~S}$ rRNA. Appl Environ Microbiol 67:172-178

Gomes NCM, Heuer H, Schönfeld J, Costa R, Mendonça-Hagler L, Smalla K (2001) Bacterial diversity of the rhizosphere of maize (Zea mays) grown in tropical soil studied by temperature gradient gel electrophoresis. Plant Soil 232:167-180

Herzberg MA, Klein DA, Coleman DC (1978) Trophic interactions in soils as they affect energy and nutrient dynamics. II. Physiological responses of selected rhizosphere bacteria. Microb Ecol 4:351-359

İnceoğlu Ö, van Overbeek LS, Falcão Salles J, van Elsas JD (2013) Normal operating range of bacterial communities in soil used for potato cropping. Appl Environ Microbiol 79:1160-1170

Kloepper JW, Beauchamp CJ (1992) A review of issues related to measuring colonization of plant roots by bacteria. Can J Microbiol 38: 1219-1232

Koch AL (1981) Growth measurement. In: Gerhardt P, Murray RGE, Costilow RN, Nester EW, Wood WA, Krieg NR, Phillips GH (eds) Manual of methods for general microbiology. American Society for Microbiology, Washington, pp 179-207 
Krutz LJ, Shaner DL, Accinelli C, Zablotowicz RM, Henry WB (2008) Atrazine dissipation in $s$-triazine-adapted and non-adapted soil from Colorado and Mississippi: implications of enhanced degradation on atrazine fate and transport parameters. J Environ Qual 37:848-857

Krutz LJ, Shaner DL, Weaver MA, Webb RMT, Zablotowicz RM, Reddy KN, Huang Y, Thomson SJ (2010) Agronomic and environmental implications of enhanced $s$-triazine degradation. Pest Manag Sci 66: 461-481

Krutz LJ, Zablotowicz RM, Reddy KN (2012) Selection pressure, cropping system, and rhizosphere proximity affect atrazine degrader populations and activity in $s$-triazine-adapted soil. Weed Sci 60: $516-524$

Lugtenberg BJJ, Dekkers L, Bloemberg GV (2001) Molecular determinants of rhizosphere colonization by Pseudomonas. Annu Rev Phytopathol 39:461-490

Lynch JM (1984) Interactions between biological processes, cultivation and soil structure. Plant Soil 76:307-318

Mandelbaum RT, Wackett LP (1996) Pseudomonas strain for degradation of s-triazines in soil and water. U.S. Patent No. 5,508,193. Washington, DC: U.S. Patent and Trademark Office

Mansfeld-Giese K, Larsen J, Bødker L (2002) Bacterial populations associated with mycelium of the arbuscular mycorrhizal fungus Glomus intraradices. FEMS Microbiol Ecol 41:133-140

Mongodin EF, Shapir N, Daugherty SC, DeBoy RT, Emerson JB, Shvartzbeyn A, Radune D, Vamathevan J, Riggs F, Grinberg V, Khouri H, Wackett LP, Nelson KE, Sadowsky MJ (2006) Secrets of soil survival revealed by the genome sequence of Arthrobacter aurescens TC1. PLoS Genet 2(12):e214. doi:10.1371/journal.pgen. 0020214

Morgante V, Lopez-Lopez A, Flores C, González M, Gonález B, Vásquez M, Roselló-Mora R, Seeger M (2010) Bioaugmentation with Pseudomonas $\mathrm{sp}$. strain MHP41 promotes simazine attenuation and bacterial community changes in agricultural soils. FEMS Microbiol Ecol 71:114-126

Newcombe DA, Crowley DE (1999) Bioremediation of atrazinecontaminated soil by repeated application of atrazine-degrading bacteria. Appl Microbiol Biotechnol 51:877-882
Polyanskaya LM, Ozerskaya SM, Kochkina GA, Ivanushkina NE, Golovchenko AV, Zvyagintsev DG (2003) The abundance and structure of the root-associated microbial complexes of two greenhouse rose cultivars. Microbiology 73:496. doi:10.1023/A: 1025061210599

Raaijmakers JM, Weller DM (2001) Exploiting the genetic diversity of Pseudomonas spp: characterization of superior root-colonizing P. fluorescens strain Q8r1-96. Appl Environ Microbiol 67:25452554

Rovira AD, Ogoshi A, McDonald HJ (1986) Characterization of isolates of Rhizoctonia solani from cereal roots in South Australia and New South Wales. Phytopathology 76:1245-1248

Sagarkar S, Bhardwaj P, Storck V, Devers-Lamrani M, MartinLaurent F, Kapley A (2016) $s$-triazine degrading bacterial isolate Arthrobacter sp. AK-YN10, a candidate for bioaugmentation of atrazine contaminated soil. Appl Microbiol Biotechnol 100:903-913

Scher FM, Zeigle JS, Kloepper JW (1984) A method for assessing the root-colonizing capacity of bacteria on maize. Can J Microbiol 30: $151-157$

Schweiger F, Tebbe C (2000) Effect of field inoculation with Sinorhizobium meliloti L33 on the composition of bacterial communities in rhizospheres of a target plant (Medicago sativa) and a nontarget plant (Chenopodium album) — linking of 16S rRNA genebased single-strand conformation polymorphism community profiles to the diversity of cultivated bacteria. Appl Env Microbiol 66: 3556-3565

Stanlake GT, Clark JB (1976) Motility is a morphogenic character in the genus Arthrobacter. J Bacteriol 127:1524-1528

Topp E (2001) A comparison of three atrazine-degrading bacteria for soil bioremediation. Biol Fertil Soils 33:529-534

Wang Q, Xie S, Hu R (2013) Bioaugmentation with Arthrobacter sp. strain DAT1 for remediation of heavily atrazine-contaminated soil. Int Biodeterior Biodegradation 77:63-67

Winogradsky S (1925) Études sur la microbiologie du sol. Sur la méthode. Ann Inst Pasteur 39:299-310 